\title{
Cochrane
}

Cochrane Database of Systematic Reviews

\section{Surgical interventions for the management of chronic groin pain after hernia repair (postherniorrhaphy inguinodynia) in adults (Protocol)}

Zwaans WAR, Koning GG, Gurusamy KS, van Kleef M, Scheltinga MRM, Roumen RMH

Zwaans WAR, Koning GG, Gurusamy KS, van Kleef M, Scheltinga MRM, Roumen RMH.

Surgical interventions for the management of chronic groin pain after hernia repair (postherniorrhaphy inguinodynia) in adults. Cochrane Database of Systematic Reviews 2017, Issue 4. Art. No.: CD012630.

DOI: 10.1002/14651858.CD012630.

www.cochranelibrary.com

Surgical interventions for the management of chronic groin pain after hernia repair (postherniorrhaphy inguinodynia) in adults (Protocol) Copyright $\odot 2017$ The Cochrane Collaboration. Published by John Wiley \& Sons, Ltd. 
TABLE OF CONTENTS

HEADER . . . . . . . . . . . . . . . . . . . . . . . . . . . . . . . . . . . . 1

ABSTRACT . . . . . . . . . . . . . . . . . . . . . . . . . . . . . . . . . . . . . . . . . .

BACKGROUND . . . . . . . . . . . . . . . . . . . . . . . . . . . . . . . . . . . . .

OBJECTIVES . . . . . . . . . . . . . . . . . . . . . . . . . . . . . . . . . . . . . . .

METHODS . . . . . . . . . . . . . . . . . . . . . . . . . . . . . . . . . . . . . .

ACKNOWLEDGEMENTS . . . . . . . . . . . . . . . . . . . . . . . . . . . . . . . . . . . . . . . .

REFERENCES . . . . . . . . . . . . . . . . . . . . . . . . . . . . . . . . . . . . . 7

APPENDICES . . . . . . . . . . . . . . . . . . . . . . . . . . . . . . . . . . . . . . 10

CONTRIBUTIONS OF AUTHORS . . . . . . . . . . . . . . . . . . . . . . . . . . . . . . . . . . . 11

DECLARATIONS OF INTEREST . . . . . . . . . . . . . . . . . . . . . . . . . . . . . . . 11

Surgical interventions for the management of chronic groin pain after hernia repair (postherniorrhaphy inguinodynia) in adults 


\title{
Surgical interventions for the management of chronic groin pain after hernia repair (postherniorrhaphy inguinodynia) in adults
}

\author{
Willem AR Zwaans ${ }^{1}$, Giel G Koning ${ }^{2}$, Kurinchi Selvan Gurusamy ${ }^{3}$, Maarten van Kleef ${ }^{4}$, Marc RM Scheltinga ${ }^{1}$, Rudi MH Roumen ${ }^{1}$ \\ ${ }^{1}$ General Surgery, Máxima Medical Center, Veldhoven, Netherlands. ${ }^{2}$ Department of Surgery, Medical Center Leeuwarden, Leeuwar- \\ den, Netherlands. ${ }^{3}$ Department of Surgery, Royal Free Campus, UCL Medical School, London, UK. ${ }^{4}$ Anaesthesiology/Pain Medicine, \\ Maastricht University Medical Center, Maastricht, Netherlands
}

Contact address: Willem AR Zwaans, General Surgery, Máxima Medical Center, Veldhoven, Netherlands.willemzwaans@gmail.com, w.zwaans@mmc.nl.

Editorial group: Cochrane Pain, Palliative and Supportive Care Group.

Publication status and date: New, published in Issue 4, 2017.

Citation: Zwaans WAR, Koning GG, Gurusamy KS, van Kleef M, Scheltinga MRM, Roumen RMH. Surgical interventions for the management of chronic groin pain after hernia repair (postherniorrhaphy inguinodynia) in adults. Cochrane Database of Systematic Reviews 2017, Issue 4. Art. No.: CD012630. DOI: 10.1002/14651858.CD012630.

Copyright (C) 2017 The Cochrane Collaboration. Published by John Wiley \& Sons, Ltd.

\begin{abstract}
A B S T R A C T
This is a protocol for a Cochrane Review (Intervention). The objectives are as follows:

To assess the efficacy and safety of surgical interventions for the management of groin pain as a consequence of previous inguinal hernia repair in adults.
\end{abstract}

\section{B A C K G R O U N D}

Inguinal hernia repair is one of the most frequently performed procedures in general surgery, with approximately 20 million repairs every year worldwide (Kingsnorth 2003). The gold standard for treating inguinal hernias is with a mesh resulting in a tensionfree repair (Simons 2009). The synthetic mesh overlaps the inguinal defect with the aim of reinforcing the abdominal wall. After the introduction of mesh for inguinal hernia repair, the number of people with recurrences has dropped drastically (Collaboration EUHT 2002). Therefore, clinical interest has switched towards chronic pain as the most important, long-term, costly and invalidating complication (Bay-Nielsen 2001; Courtney 2002).

\section{Description of the condition}

Chronic postoperative inguinal pain (postherniorrhaphy inguinodynia or CPIP) is defined by the International Association for the Study of Pain as "pain beyond three months after inguinal hernia surgery” (IASP 1986). The incidence of chronic pain following repair with use of a mesh, like the Lichtenstein technique, varies between 5\% and 54\% (Poobalan 2003; Loos 2007a; Kehlet 2008), with a pooled incidence of $11 \%$ to $16.8 \%$ (Perkins 2000 ; Nienhuijs 2007; Simons 2009; Koning 2013). Some $2 \%$ to $6 \%$ of patients experience significant restrictions in social and daily activities as a consequence of CPIP, leading to an impairment of health status or 'quality of life' (QoL) (Callesen 1999; Poobalan 2001; Bozuk 2003; Mikkelsen 2004; Aasvang 2005a). Earlier studies reported that people undergoing surgery for recurrent inguinal

Surgical interventions for the management of chronic groin pain after hernia repair (postherniorrhaphy inguinodynia) in adults 
hernias had a fourfold higher probability of developing moderate to severe pain as compared to those undergoing primary repairs (Callesen 1999). Although the development of endoscopic techniques for hernia repair has resulted in lower pain incidences $(6 \%$ to $12.4 \%$ (Aasvang 2005a; Koning 2013)), CPIP remains a severe (and probably the most incapacitating) complication following inguinal hernia surgery.

CPIP is generally classified as neuropathic and non-neuropathic (inflammatory or nociceptive) pain. Neuropathic postherniorrhaphy pain can be a result of nerve entrapment by the inserted mesh or direct damage to inguinal nerves during surgery (Perkins 2000; Kehlet 2006; Loos 2007b). Other proposed pathological mechanisms involved in chronic neuropathic pain include traumatic neuroma formation (leading to ectopic excitability, in approximately $12 \%$ (Zwaans 2015)), perineural scar tissue development, and entrapment of nerves due to fibrosis and sensitization (Amid 2004a; Chaparro 2013). The principal clinical characteristics of neuropathic pain are a sharp, burning or 'shooting' sensation which is progressive after repetitive stimulation. Paraesthesia ('tingling', 'crawling', or electrical sensations) and dysaesthesia (spontaneous or evoked unpleasant abnormal sensation) with radiation towards the associated skin area of the involved inguinal nerve are often reported. Depending on the affected nerve, pain may radiate towards the upper medial thigh (ilioinguinal nerve), suprapubic region (iliohypogastric nerve) or the genitals or ventral upper leg (genitofemoral nerve). Furthermore, patients may complain of motor deficits in the damaged nerve territory. During physical examination, a neuropathic postherniorrhaphy pain is characterised by the presence of a point of maximum pain that is covered by a somewhat larger skin area having abnormal sensation. Pain sensation is disproportionally painful (hyperalgesic) when this skin is pinched. Positive sensory abnormalities such as allodynia, hyperalgesia and hyperpathia or negative neurophysiologic phenomena including hypoaesthesia and hypoalgesia may support the diagnosis (Baron 2006). Furthermore, high scores on a neuropathic pain diagnostic questionnaire (DN4) may be helpful in differentiating this specific type from non-neuropathic pain (Bouhassira 2005).

On the other hand, non-neuropathic chronic postherniorrhaphy pain may be a consequence of a mesh- or suture-induced (inflammatory) reaction of the inguinal area. A mesh-related response is usually ongoing for several months following inguinal hernia repair (Aasvang 2005a). These include inflammatory-related pain syndromes such as periostitis of the pubic bone. Since synthetic meshes have the tendency to wrinkle and crease over time (Amid $2004 \mathrm{~b}$ ), the formation of a so-called meshoma can also lead to pain by a volume effect or by mechanical pressure on surrounding structures. People with non-neuropathic postherniorrhaphy pain report a throbbing or nagging pain located in a non-neuroanatomical area (Rasmussen 2004). Local pain increases if pressure is applied to either the mesh (mesh-related), the pubic bone (periostitis) or the funiculus (funiculodynia) (Loos 2007b). Pain related to the mesh itself is usually suspected when people complain of a 'foreign body' sensation or feeling of tightness in the groin area. The pain can be aggravated by driving or leg crossing, whereas hip extension may relieve the pain (Zwaans 2017). During physical examination, deep palpation along the Poupart's ligament and lack of sensory loss may help the physician in the diagnosis of a mesh-related type of postherniorrhaphy pain. A more diffuse pain is usually found whereas sometimes the meshoma can be felt in non-obese individuals (Zwaans 2017).

The exact diagnosis of chronic postherniorrhaphy pain mainly depends on concise history-taking and an extensive physical examination. A diagnostic local nerve block may aid in confirming the diagnosis (Lichtenstein 1988; Loos 2010b). However, it must be recognized that differentiation between neuropathic and non-neuropathic pain is often difficult, if not sometimes impossible as objective diagnostic measurements are currently lacking (Kehlet 2013). In addition, a combined pain syndrome entailing neuropathic and nociceptive elements is not uncommon following hernia repair. Dysejaculation (Aasvang 2008; Bischoff 2012; Verhagen 2016) or orchialgia (Masarani 2003; Chen 2015) may be observed during both types of pain.

Risk factors for CPIP have been investigated extensively. Known factors to increase the risk of chronic pain include a high preoperative pain intensity (Franneby 2006; Simons 2009; Pierides 2016), the preoperative presence of chronic pain conditions (Simons 2009), female gender (Bay-Nielsen 2001; Mori 2001; Aasvang 2005a; Simons 2009), young age (Franneby 2006; Simons 2009; Pierides 2016), general anaesthesia (Ozgun 2002; Joshi 2012; Zwaans 2015), anterior open approach (versus laparoscopic repair) (Franneby 2006; Nienhuijs 2007; Simons 2009), incomplete identification of all three inguinal nerves (Alfieri 2006; Simons 2009), use of a heavy-weighted polypropylene mesh for repair (Nienhuijs 2007; Simons 2009), severe immediate postoperative pain (Kehlet 2006; Simons 2009; Aasvang 2010), and postoperative complications (Franneby 2006; Pierides 2016).

However, it must be appreciated that the relative contribution of these factors on CPIP is unknown. Moreover, psychological factors including expectations, anxiety, depression, past memories and social environment are recognized to play a role in the experience and the development of chronic pain in general (Tasmuth 1996; Turk 1996; Courtney 2002; Kehlet 2006).

Although some studies suggest that immediate postherniorrhaphy pain may diminish over time (Grant 2004; Nienhuijs 2007), the majority of painful patients may develop a chronic character of postherniorrhaphy pain. First-line management of CPIP is pharmacological, using conventional analgesics or by peripheral nerve blocks with local anaesthetics (whether or not combined with corticosteroids). It is recognized that adequate pharmacological pain management is important in the acute phase as a means to minimise the chance of conversion to chronic pain (Werner 2014a; Werner 2014b). However, if conservative treatments are to no avail, more invasive therapies may be considered. Anaesthesio- 
logic techniques including transcutaneous electric nerve stimulation (TENS), pulsed radiofrequency (PRF), nerve root blocks or dorsal root ganglion stimulation may all offer pain relief. Surgical interventions are usually considered as a last option once pain is recalcitrant.

\section{Description of the intervention}

Surgical therapies to relieve CPIP (remedial surgery) can be performed using an open approach (Starling 1989; Heise 1998; Amid 2002; Deysine 2002; Amid 2004a; Aasvang 2005b; Loos 2010a; Verhagen 2016); or an endoscopic approach (Krahenbuhl 1997; Giger 2009; Chen 2013). Two major types of remedial surgery can be distinguished. The rationale for the choice of remedial surgery mainly depends on the assumed cause of the postherniorrhaphy pain. Resection of the inguinal nerves, either by a tailored approach or as triple neurectomy including all inguinal nerves, is the most performed remedial surgical technique for (neuropathic) CPIP (Lange 2015). The number of neurectomies depends on the preference of the attending surgeon.

A second type of remedial surgery is the removal of the surgical mesh (meshectomy), either partial or complete. This surgical procedure can be considered when the mesh is implicated as the origin of non-neuropathic postherniorrhaphy pain. A meshectomy may be complex and a salvage technique, since surgery in fibrotic tissue may increase the chances of complications considerably. Moreover, the posterior wall or the inguinal canal may require reinforcement to avoid looming recurrent hernias. This can be done by a synchronous or metachronous new mesh insertion (Keller 2008) or by a tension repair using own body material (Koopmann 2011; Zwaans 2017).

Other types of remedial surgery may entail removal of fixating devices such as sutures or staples. A release of the funiculus (funiculolysis) may be helpful in a subgroup of patients, as is an orchiectomy. All of these procedures are infrequently performed but may offer pain relief in highly selective groups of patients.

\section{How the intervention might work}

Surgical interventions of postherniorrhaphy pain are guided by symptomatology, clinical findings and the type of primary hernia repair. When nerve lesions or other neuropathic triggers responsible for the inguinodynia are suspected, a neurectomy of one or more of the affected nerves is considered. By removing the driving force of the neuropathy, the pain is assumed to fade out (Amid 2002). Inguinal pain is frequently replaced by loss of normal skin sensation. However, central sensitization of pain may corrupt this phenomenon. The same accounts for a meshectomy or removal of fixation devices. By eliminating the origin, the nociceptive pain diminishes over time (Kehlet 2006). It must be appreciated that the intervention also depends on intraoperative findings. For ex- ample, when a nociceptive source of pain is suspected, but a neuroma is found intraoperatively, this neuroma requires removal. For men undergoing surgery, funiculolysis can be performed. Funiculolysis (dissection of the funiculus from its surrounding tissue) includes the release of the spermatic cord in order to attenuate congestion of the testicular vein or remove extensive fibrotic tissue cornering structures like the vas deferens. Orchiectomy is occasionally considered once all other options have failed and the cause of nociceptive pain is thought to be caused by the testicle itself (Chen 2015).

\section{Why it is important to do this review}

The proper management of postherniorrhaphy pain is a challenging obstacle and clearly needs systematic reviewing of the available literature. Although remedial surgeries are performed in specialised centres by experienced and well-trained surgeons, there is a necessity for evidence-based practice to rationally evaluate safety and efficacy of these interventions. Although a limited number of reviews have been published over the years, uncertainty remains and no firm conclusions can be drawn as also stipulated by international guidelines on hernia surgery (Simons 2009). If surgical therapies are more effective than conservative therapies, a more prominent step in the algorithm would be appropriate to avoid unnecessary long intervals of postherniorrhaphy pain, especially since it is thought that if pain is not treated effectively in the early stages then with time it may become more difficult to treat.

\section{O B J E C T IVES}

To assess the efficacy and safety of surgical interventions for the management of groin pain as a consequence of previous inguinal hernia repair in adults.

\section{METHODS}

\section{Criteria for considering studies for this review}

\section{Types of studies}

We will include studies if they are randomised controlled trials (RCTs) with double-blind assessment of participant outcomes. We require full journal publication, with the exception of online clinical trial results, summaries of otherwise unpublished clinical trials and abstracts with sufficient data for analysis. We will exclude studies that were non-randomised, studies of experimental pain, case reports and clinical observations. 


\section{Types of participants}

Adults aged 18 years and older who underwent surgical interventions for chronic (> 3 months) groin pain following inguinal hernia repair will be considered. If only a subset of randomised participants is relevant in a study, the subgroup of interest will be used provided that data are specified separately.

\section{Types of interventions}

The experimental interventions are surgical procedures aimed at reducing postherniorrhaphy groin pain, including (but not limited to): (triple or tailored) neurectomy; (partial or complete) meshectomy; funicular release; removal of endoscopic staples or (fixating) sutures; and orchiectomy.

\section{Control intervention}

All conservative treatment regimens including (but not limited to): sham surgery; analgesics (NSAIDs, opioids, antidepressants, gabapentin, pregabalin, antipsychotics, topical lidocaine, topical capsaicin); transcutaneous electric nerve stimulation (TENS); (pulsed) radiofrequency (PRF); onabotulinumtoxinA (Botox); nerve blocks with local anaesthetics; nerve blocks with local anaesthetics and corticosteroids; nerve root blocks; dorsal root ganglion stimulation; cryotherapy; and psychological therapies.

\section{Types of outcome measures}

\section{Primary outcomes}

- Pain as a continuous outcome using the Numerical Rating Scale (NRS) or Visual Analogue Scale (VAS).

- Pain reduction as a binary outcome (yes or no).

- Adverse events (including hematoma, seroma, wound infection, recurrences, testicular atrophy, vascular injury, visceral injury) at any time point following the intervention. Severity of adverse events will be graded using the validated Clavien-Dindo classification of surgical complications (Clavien 1992; Dindo 2004; Clavien 2009).

Timing of these outcomes will be at different time points following the intervention: 3 months, 6 months, 12 months and 24 months.

- Treatment-related severe adverse events (death, permanent or significant invalidity due to the procedure) at any time point following the intervention.

\section{Secondary outcomes}

- Participant-reported pain relief of $30 \%$ or greater as a binary outcome at 3 months, 6 months, 12 months and 24 months following the procedure.
- Participant-reported pain relief of $50 \%$ or greater as a binary outcome at 3 months, 6 months, 12 months and 24 months following the procedure.

- Worsening of pain (participant self-reported) as a binary outcome at 3 months, 6 months, 12 months and 24 months following the procedure.

- QoL outcomes (participant self-reported or health status reports).

\section{Search methods for identification of studies}

\section{Electronic searches}

We will search the following databases.

- Cochrane Central Register of Controlled Trials

(CENTRAL) in the Cochrane Library.

- MEDLINE (via PubMED).

- Embase (OVID).

We will use MeSH or equivalent and text word terms. We will impose no language restrictions. We will tailor searches to individual databases.

The search strategy for MEDLINE is shown in Appendix 1.

\section{Searching other resources}

We will check reference lists of reviews and retrieved articles for additional studies. We will search the following clinical trial registers for identifying relevant unpublished trials: the metaRegister of Controlled Trials (mRCT) (controlledtrials.com), ClinicalTrials.gov (clinicaltrials.gov), the Dutch Trial Register (trialregister.nl) and the World Health Organization (WHO) International Clinical Trials Registry Platform (ICTRP) (apps.who.int/trialsearch).

\section{Data collection and analysis}

\section{Selection of studies}

Two authors (WARZ, GGK) will determine eligibility of studies by reading each abstract as identified by the electronic search strategy. They will exclude studies that clearly do not satisfy eligibility criteria and will document these in a separate table. We will obtain full copies of the remaining studies to assess eligibility. Two authors will separately read the papers and will concur on the study selection. If consensus fails, they will consult a third reviewer. Studies will be blinded before assessment. If only a subset of randomised participants in a certain study is relevant but the subgroup of interest involves the majority of participants, these data will be used in the present systematic review. We will resolve 
any problems during this part of the reviewing process by consensus.

A flowchart of selection of studies according to the PRISMA statement will provide insight into the screening process (Liberati 2009).

\section{Data extraction and management}

Two authors will independently extract data from the included studies using a data collection form. They will resolve any discrepancies by consensus. If no consensus is reached, they will consult a third author to overcome these issues.

We will extract the following data: country of origin; language; period of approval (in published studies); population characteristics (including age, sex ratio, type of primary inguinal hernia repair); sample size; inclusion and exclusion criteria; type of surgical intervention; type of anaesthesia used during surgical intervention; type of control; primary and secondary outcomes as described above including time of assessment; delay between primary repair and intervention; pre-intervention and post-intervention pain assessments; type of pain assessment (including pain scale or quantitative sensory testing (QST)); adverse effects; industry sponsorship; conflict of interest statements.

We will perform a separate analysis for the different types of surgical intervention. We will perform additional analyses for gender, type of primary hernia repair, primary open versus primary endoscopic inguinal hernia repair, and type of anaesthesia.

If we identify a study that has more than two intervention arms, we will only include the intervention and control groups that meet the eligibility criteria in the analysis. We will contact the corresponding authors of the individual trials if there are any unclear or missing data.

\section{Assessment of risk of bias in included studies}

Two authors (WARZ, GGK) will assess the risk of bias in individual studies, without masking for trial names, using the criteria outlined in the Cochrane Handbook for Systematic Reviews of Interventions (Higgins 2011). Disagreement between the two authors will be resolved by consensus. If they cannot reach consensus, they will consult a third author to overcome this issue. We will complete a 'Risk of bias' table for each included study using the 'Risk of bias' tool in RevMan (Review Manager 2014).

We will assess the following for each study.

1. Random sequence generation (checking for possible selection bias). We will assess the method used to generate the allocation sequence as: low risk of bias (any truly random process); unclear risk of bias (method used to generate sequence not clearly stated). Studies using a non-random process will be excluded.

2. Allocation concealment (checking for possible selection bias). The method used to conceal allocation to interventions prior to assignment determines whether intervention allocation could have been foreseen in advance of (or during) recruitment, or changed after assignment. We will assess the methods as: low risk of bias (e.g. telephone or central randomisation; consecutively numbered sealed opaque envelopes); unclear risk of bias (method not clearly stated). Studies that do not conceal allocation will be excluded.

3. Blinding of outcome assessment (checking for possible detection bias). We will assess the methods used to blind study participants and outcome assessors from knowledge of which intervention a participant received. We will assess the methods as: low risk of bias (study states that it was blinded and describes the method used to achieve blinding); unclear risk of bias (study states that it was blinded but does not provide an adequate description of how it was achieved). Studies that were not double-blind will be excluded.

4. Selective reporting (checking for reporting bias). We will assess whether primary and secondary outcome measures were pre-specified and whether these were consistent with those reported as: low risk of bias (i.e. $<10 \%$ of participants did not complete the study); unclear risk of bias (used 'last observation carried forward' analysis); or high risk of bias (used completer analysis).

5. Incomplete outcome data (checking for possible attrition bias due to the amount, nature and handling of incomplete outcome data). We will assess the methods used to deal with incomplete data as: low risk $(<10 \%$ of participants did not complete the study and/or used 'baseline observation carried forward' analysis); unclear risk of bias (used 'last observation carried forward' analysis); high risk of bias (used 'completer' analysis).

6. Size of study (checking for possible biases confounded by small size). We will assess studies as being at low risk of bias $(\geq$ 200 participants per treatment arm); unclear risk of bias (50 to 199 participants per treatment arm); high risk of bias $(<50$ participants per treatment arm).

\section{Measures of treatment effect}

We will pool dichotomous data to calculate relative risk (RR) with corresponding $95 \%$ confidence intervals (CI). We will calculate the mean difference (MD) or standardised mean differences (SMD) with $95 \%$ CI for continuous variables. We will present continuous primary outcome measures (QoL outcomes) as MD or SMDs, in equal or different pain assessments, respectively. We will consider a $P$ value of less than 0.05 as statistically significant for both dichotomous and continuous data. We will present treatment-related (severe) adverse events as descriptive statistics.

\section{Unit of analysis issues}

The unit of analysis will be individual participants. 


\section{Dealing with missing data}

We will use intention-to-treat (ITT) analysis where the ITT population consists of participants who were randomised, underwent a conservative treatment at least once, and provided at least one post-baseline assessment. Missing participants will be assigned zero improvement wherever possible.

We will make at least two attempts to contact study authors for missing data by email.

\section{Assessment of heterogeneity}

We will assess heterogeneity by calculation of inconsistency statistics $\left(\mathrm{I}^{2}\right)$. We will investigate the significance of potential heterogeneity by the $\mathrm{Chi}^{2}$ test, considering $\mathrm{P}$ values of less than 0.1 as significant. We will perform meta-analyses using both the fixedeffect and random-effects model. If equivalent results are provided with these models, we will provide the results of the random-effects model. Otherwise, we will report the results of both models. In case heterogeneity is present between studies, a regression analysis will be performed to analyse potential confounding factors. These include type of surgical intervention, sex ratio, age and type of anaesthesia.

\section{Assessment of reporting biases}

We will not depend on what the authors of the original studies chose to report or not, though clearly difficulties will arise in studies failing to report any dichotomous results. We will extract and use continuous data, which probably will reflect efficacy and utility poorly, and may be useful for illustrative purposes only.

We will assess publication bias using a method designed to detect the amount of unpublished data with a null effect required to make any result clinically irrelevant (Moore 2008), when more than 10 studies include an outcome measure. We will exclude studies using an extended population of a previously performed study whereas the latest study will be used for analyses.

\section{Data synthesis}

We will perform a meta-analysis, according to the methods described in the Cochrane Handbook for Systematic Reviews of Interventions if possible (Higgins 2011), using Review Manager 5 software (Review Manager 2014). We will generate forest plots to illustrate the pooled outcome measures when these are judged to be sufficiently similar. Characteristics of included studies will be depicted in tables, whereas a plot will illustrate the risk of bias in the included studies. We will produce 'Summary of findings' tables, summarizing the results of the trials with a low risk of bias and for all trials.

\section{Grading of evidence}

Two review authors (WARZ, GGK) will independently rate the quality of each outcome. We will use the GRADE system to rank the quality of the evidence using the GRADEprofiler Guideline Development Tool software (GRADEpro GDT 2015), and the guidelines provided in Chapter 12.2 of the Cochrane Handbook for Systematic Reviews of Interventions (Higgins 2011).

The GRADE approach uses five considerations (study limitations; consistency of effect; imprecision; indirectness; and publication bias) to assess the quality of the body of evidence for each outcome. The GRADE system uses the following criteria for assigning the grade of evidence.

- High: we are very confident that the true effect lies close to that of the estimate of the effect.

- Moderate: we are moderately confident in the effect estimate; the true effect is likely to be close to the estimate of effect, but there is a possibility that it is substantially different;

- Low: our confidence in the effect estimate is limited; the true effect may be substantially different from the estimate of the effect.

- Very low: we have very little confidence in the effect estimate; the true effect is likely to be substantially different from the estimate of effect.

We will decrease grade rating by one $(-1)$ or two $(-2)$ if we identify:

- serious $(-1)$ or very serious $(-2)$ limitation to study quality;

- important inconsistency $(-1)$;

- some $(-1)$ or major $(-2)$ uncertainty about directness;

- imprecise or sparse data $(-1)$;

- high probability of reporting bias $(-1)$.

\section{'Summary of findings' table}

We plan to include a 'Summary of findings' table to present the main findings for comparison of surgical and conservative interventions in a transparent and simple tabular format. In particular, we will include key information concerning the quality of evidence, the magnitude of effect of the interventions examined, and the sum of available data on all primary outcomes and participantreported pain relief of greater than $50 \%$, participant-reported pain relief of greater than $30 \%$, and worsening of pain.

\section{Subgroup analysis and investigation of heterogeneity}

The following subgroup analyses will be conducted, if sufficient data are available.

- Type of surgical intervention (neurectomy, removal of endoscopic staples or fixating sutures, mesh removal, funicular release, orchiectomy).

- Tailored versus triple neurectomy.

- Male versus female participants. 
- Primary open versus primary endoscopic inguinal hernia repair.

- Type of anaesthesia (local, spinal, general).

\section{Sensitivity analysis}

We will perform a sensitivity analysis on the risk of bias, if adequate data are available. We will use the sensitivity analysis to investigate the effects on outcomes when including or excluding studies with a high risk of bias. The sensitivity analysis will be performed according to the guidelines in the Cochrane Handbook for Systematic Reviews of Interventions (Higgins 2011).

\section{Trial sequential analysis}

We will perform a trial sequential analysis (TSA) for any outcome measures if a significant difference is found and more than 2000 randomised participants are identified for the particular outcome. This statistical analysis is used to assess the risk of type I errors in the conducted meta-analysis of cumulated data. Furthermore, it may provide additional information on the number of participants needed in future studies.
The alpha and beta errors used for TSA will be set at 5\% and 20\% respectively. We will use a relative risk reduction of $25 \%$ and the control group portion as found in the review for TSA.

\section{ACKNOWLEDGEMENTS}

The members of this review team would like to thank Eugenie Delvaux (medical information specialist) for her contribution to the development of the search strategy.

We thank the reviewers, Dr. Alimi F. Mustapha, Dr. Jane Quinlan and Dr. Alfredo Covarrubias-Gomez, for their comments.

Cochrane Review Group funding acknowledgement: the National Institute for Health Research (NIHR) is the largest single funder of the Cochrane Pain, Palliative and Supportive Care Review Group $(\mathrm{PaPaS})$. Disclaimer: the views and opinions expressed herein are those of the authors and do not necessarily reflect those of the NIHR, National Health Service (NHS), or the Department of Health.

\section{R E F E R E N C E S}

\section{Additional references}

\section{Aasvang 2005a}

Aasvang E, Kehlet H. Chronic postoperative pain: the case of inguinal herniorrhaphy. British Journal of Anaesthesia 2005;95(1):69-76. [DOI: 10.1093/bja/aei019]

\section{Aasvang 2005b}

Aasvang E, Kehlet H. Surgical management of chronic pain after inguinal hernia repair. British Journal of Surgery 2005; 92(7):795-801. [DOI: 10.1002/bjs.5103]

\section{Aasvang 2008}

Aasvang EK, Kehlet H. Postherniotomy dysejaculation: Successful treatment with mesh removal and nerve transection. Hernia 2008;12(6):645-7. [DOI: 10.1007/ s10029-008-0373-1]

Aasvang 2010

Aasvang EK, Gmaehle E, Hansen JB, Gmaehle B, Forman JL, Schwarz J, et al. Predictive risk factors for persistent postherniotomy pain. Anesthesiology 2010;112(4):957-69. [DOI: 10.1097/ALN.0b013e3181d31ff8]

\section{Alfieri 2006}

Alfieri S, Rotondi F, Di Giorgio A, Fumagalli U, Salzano A, Di Miceli D, et al. Influence of preservation versus division of ilioinguinal, iliohypogastric, and genital nerves during open mesh herniorrhaphy: prospective multicentric study of chronic pain. Annals of Surgery 2006;243(4):553-8. [DOI: 10.1097/01.sla.0000208435.40970.00]

\section{Amid 2002}

Amid PK. A 1-stage surgical treatment for postherniorrhaphy neuropathic pain: triple neurectomy and proximal end implantation without mobilization of the cord. Archives of Surgery 2002;137(1):100-4.

\section{Amid 2004a}

Amid PK. Causes, prevention, and surgical treatment of postherniorrhaphy neuropathic inguinodynia: triple neurectomy with proximal end implantation. Hernia 2004; 8(4):343-9. [DOI: 10.1007/s10029-004-0247-0]

\section{Amid 2004b}

Amid PK. Radiologic images of meshoma: a new phenomenon causing chronic pain after prosthetic repair of abdominal wall hernias. Archives of Surgery 2004;139(12): 1297-8. [DOI: 10.1001/archsurg.139.12.1297]

Baron 2006

Baron R. Mechanisms of disease: neuropathic pain--a clinical perspective. Nature Clinical Practice Neurology 2006;2(2):95-106. [DOI: 10.1038/ncpneuro0113]

\section{Bay-Nielsen 2001}

Bay-Nielsen M, Perkins FM, Kehlet H. Pain and functional impairment 1 year after inguinal herniorrhaphy: a nationwide questionnaire study. Annals of Surgery 2001;233 (1):1-7.

\section{Bischoff 2012}

Bischoff JM, Linderoth G, Aasvang EK, Werner MU, Kehlet H. Dysejaculation after laparoscopic inguinal herniorrhaphy: A nationwide questionnaire study. Surgical 
Endoscopy and Other Interventional Techniques 2012;26(4):

979-83. [DOI: 10.1007/s00464-011-1980-y]

Bouhassira 2005

Bouhassira D, Attal N, Alchaar H, Boureau F, Brochet

$\mathrm{B}$, Bruxelle J, et al. Comparison of pain syndromes associated with nervous or somatic lesions and development of a new neuropathic pain diagnostic questionnaire (DN4). Pain 2005;114(1-2):29-36. [DOI: 10.1016/ j.pain.2004.12.010]

Bozuk 2003

Bozuk M, Schuster R, Stewart D, Hicks K, Greaney G, Waxman K. Disability and chronic pain after open mesh and laparoscopic inguinal hernia repair. American Journal of Surgery 2003;69(10):839-41.

\section{Callesen 1999}

Callesen T, Bech K, Kehlet H. Prospective study of chronic pain after groin hernia repair. British Journal of Surgery 1999;86(12):1528-31. [DOI: 10.1046/ j.1365-2168.1999.01320.x]

\section{Chaparro 2013}

Chaparro LE, Smith SA, Moore RA, Wiffen PJ, Gilron I. Pharmacotherapy for the prevention of chronic pain after surgery in adults. Cochrane Database of Systematic Reviews 2013, Issue 7. [DOI: 10.1002/ 14651858.CD008307.pub2]

Chen 2013

Chen DC, Hiatt JR, Amid PK. Operative management of refractory neuropathic inguinodynia by a laparoscopic retroperitoneal approach. JAMA Surgery 2013;148(10): 962-7. [DOI: 10.1001/jamasurg.2013.3189]

Chen 2015

Chen DC, Amid PK. Persistent orchialgia after inguinal hernia repair: diagnosis, neuroanatomy, and surgical management: Invited comment to: Role of orchiectomy in severe testicular pain and inguinal hernia surgery: audit of Finnish patient insurance centre. Hernia 2015;19(1):61-3. [DOI: $10.1007 / s 10029-013-1172-x]$

\section{Clavien 1992}

Clavien PA, Sanabria JR, Strasberg SM. Proposed classification of complications of surgery with examples of utility in cholecystectomy. Surgery 1992;111(5):518-26.

\section{Clavien 2009}

Clavien PA, Barkun J, de Oliveira ML, Vauthey JN, Dindo D, Schulick RD, et al. The Clavien-Dindo classification of surgical complications: five-year experience. Annals of Surgery 2009;250(2):187-96. [DOI: 10.1097/ SLA.0b013e3181b13ca2]

Collaboration EUHT 2002

Collaboration EUHT. Repair of groin hernia with synthetic mesh: meta-analysis of randomized controlled trials. Annals of Surgery 2002;235(3):322-32.

\section{Courtney 2002}

Courtney CA, Duffy K, Serpell MG, O’Dwyer PJ. Outcome of patients with severe chronic pain following repair of groin hernia. British Journal of Surgery 2002;89(10):1310-14. [DOI: 10.1046/j.1365-2168.2002.02206.x]

Deysine 2002

Deysine M, Deysine GR, Reed WP Jr. Groin pain in the absence of hernia: a new syndrome. Hernia 2002;6(2): 64-7.

Dindo 2004

Dindo D, Demartines N, Clavien PA. Classification of surgical complications: a new proposal with evaluation in a cohort of 6336 patients and results of a survey. Annals of Surgery 2004;240(2):205-13.

\section{Franneby 2006}

Franneby U, Sandblom G, Nordin P, Nyren O, Gunnarsson $\mathrm{U}$. Risk factors for long-term pain after hernia surgery. Annals of Surgery 2006;244(2):212-19. [DOI: 10.1097/ 01.sla.0000218081.53940.01]

\section{Giger 2009}

Giger U, Wente MN, Buchler MW, Krahenbuhl S, Lerut J, Krahenbuhl L. Endoscopic retroperitoneal neurectomy for chronic pain after groin surgery. British Journal of Surgery 2009;96(9):1076-81. [DOI: 10.1002/bjs.6623]

\section{GRADEpro GDT 2015 [Computer program]}

McMaster University (developed by Evidence Prime, Inc.). GRADEpro Guideline Development Tool. Available from www.gradepro.org, 2015.

\section{Grant 2004}

Grant AM, Scott NW, O'Dwyer PJ. Five-year follow-up of a randomized trial to assess pain and numbness after laparoscopic or open repair of groin hernia. British Journal of Surgery 2004;91(12):1570-4. [DOI: 10.1002/bjs.4799]

\section{Heise 1998}

Heise CP, Starling JR. Mesh inguinodynia: a new clinical syndrome after inguinal herniorrhaphy?. Journal of the American College of Surgeons 1998;187(5):514-18.

\section{Higgins 2011}

Higgins JPT, Green S (editors). Cochrane Handbook for Systematic Reviews of Interventions Version 5.1.0 (updated March 2011). The Cochrane Collaboration, 2011. Available from handbook.cochrane.org.

\section{IASP 1986}

International Association for the Study of Pain, Subcommittee on Taxonomy. Classification of chronic pain. Descriptions of chronic pain syndromes and definitions of pain terms. Pain. Supplement 1986;3:S1-226.

\section{Joshi 2012}

Joshi GP, Rawal N, Kehlet H, Bonnet F, Camu F, Fischer $\mathrm{HB}$, et al. Evidence-based management of postoperative pain in adults undergoing open inguinal hernia surgery. British Journal of Surgery 2012;99(2):168-85. [DOI: 10.1002/bjs.7660]

\section{Kehlet 2006}

Kehlet H, Jensen TS, Woolf CJ. Persistent postsurgical pain: risk factors and prevention. Lancet 2006;367(9522): 1618-25. [DOI: 10.1016/S0140-6736(06)68700-X] 


\section{Kehlet 2008}

Kehlet H. Chronic pain after groin hernia repair. British Journal of Surgery 2008;95(2):135-6. [DOI: 10.1002/ bjs.6111]

\section{Kehlet 2013}

Kehlet H, Roumen RM, Reinpold W, Miserez M. Invited commentary: Persistent pain after inguinal hernia repair: what do we know and what do we need to know?. Hernia 2013;17(3):293-7. [DOI: 10.1007/s10029-013-1109-4]

\section{Keller 2008}

Keller JE, Stefanidis D, Dolce CJ, Iannitti DA, Kercher KW, Heniford BT. Combined open and laparoscopic approach to chronic pain after inguinal hernia repair. American Surgeon 2008;74(8):695-700.

\section{Kingsnorth 2003}

Kingsnorth A, LeBlanc K. Hernias: inguinal and incisional. Lancet 2003;362(9395):1561-71. [DOI: 10.1016/ S0140-6736(03)14746-0]

Koning 2013

Koning GG, Wetterslev J, van Laarhoven CJ, Keus F. The totally extraperitoneal method versus Lichtenstein's technique for inguinal hernia repair: a systematic review with meta-analyses and trial sequential analyses of randomized clinical trials. PLoS One 2013;8(1):e52599. [DOI: 10.1371/journal.pone.0052599]

\section{Koopmann 2011}

Koopmann MC, Yamane BH, Starling JR. Long-term follow-up after meshectomy with acellular human dermis repair for postherniorrhaphy inguinodynia. Archives of Surgery 2011;146(4):427-31. [DOI: 10.1001/ archsurg.2011.49]

Krahenbuhl 1997

Krahenbuhl L, Striffeler H, Baer HU, Buchler MW. Retroperitoneal endoscopic neurectomy for nerve entrapment after hernia repair. British Journal of Surgery 1997;84(2):216-19.

\section{Lange 2015}

Lange JF, Kaufmann R, Wijsmuller AR, Pierie JP, Ploeg RJ, Chen DC, et al. An international consensus algorithm for management of chronic postoperative inguinal pain. Hernia 2015;19(1):33-43. [DOI: 10.1007/s10029-014-1292-y]

\section{Liberati 2009}

Liberati A, Altman DG, Tetzlaff J, Mulrow C, Gotzsche PC, Ioannidis JP, et al. The PRISMA statement for reporting systematic reviews and meta-analyses of studies that evaluate health care interventions: explanation and elaboration. Journal of Clinical Epidemiology 2009;62(10):e1-34. [DOI: 10.1016/j.jclinepi.2009.06.006]

\section{Lichtenstein 1988}

Lichtenstein IL, Shulman AG, Amid PK, Montllor MM. Cause and prevention of postherniorrhaphy neuralgia: a proposed protocol for treatment. American Journal of Surgery 1988;155(6):786-90.

\section{Loos 2007a}

Loos MJ, Roumen RM, Scheltinga MR. Chronic sequelae of common elective groin hernia repair. Hernia 2007;11(2): 169-73. [DOI: 10.1007/s10029-007-0196-5]

\section{Loos 2007b}

Loos MJ, Roumen RM, Scheltinga MR. Classifying postherniorrhaphy pain syndromes following elective inguinal hernia repair. World Journal of Surgery 2007;31(9):1760-7. [DOI: 10.1007/s00268-007-9121-4]

Loos 2010a

Loos MJ, Scheltinga MR, Roumen RM. Tailored neurectomy for treatment of postherniorrhaphy inguinal neuralgia. Surgery 2010;147(2):275-81. [DOI: 10.1016/ j.surg.2009.08.008]

\section{Loos 2010b}

Loos MJ, Verhagen T, Scheltinga MR, Roumen RM. A randomised controlled trial of injection therapy versus neurectomy for post-herniorrhaphy inguinal neuralgia: rationale and study design. Hernia 2010;14(6):593-7. [DOI: 10.1007/s10029-010-0697-5]

\section{Masarani 2003}

Masarani M, Cox R. The aetiology, pathophysiology and management of chronic orchialgia. British Journal of Urology International 2003;91(5):435-7.

\section{Mikkelsen 2004}

Mikkelsen T, Werner MU, Lassen B, Kehlet H. Pain and sensory dysfunction 6 to 12 months after inguinal herniotomy. Anesthesia and Analgesia 2004;99(1):146-51.

Moore 2008

Moore RA, Barden J, Derry S, McQuay HJ. Managingpotential publication bias. In: McQuay HJ, Kalso E, Moore RA editor(s). Systematic Reviews in Pain Research:Methodology Refined. Seattle: IASP Press, 2008: 15-24. [ISBN: 978-0-931092-69-5]

Mori 2001

Mori T, Souda S, Nezu R, Yoshikawa Y. Results of performing mesh plug repair for groin hernias. Surgery Today 2001;31(2):129-32. [DOI: 10.1007/s005950170196]

\section{Nienhuijs 2007}

Nienhuijs S, Staal E, Strobbe L, Rosman C, Groenewoud $\mathrm{H}$, Bleichrodt R. Chronic pain after mesh repair of inguinal hernia: a systematic review. American Journal of Surgery 2007;194(3):394-400. [DOI: 10.1016/ j.amjsurg.2007.02.012]

\section{Ozgun 2002}

Ozgun H, Kurt MN, Kurt I, Cevikel MH. Comparison of local, spinal, and general anaesthesia for inguinal herniorrhaphy. European Journal of Surgery 2002;168(8-9): 455-9. [DOI: 10.1080/110241502321116442]

\section{Perkins 2000}

Perkins FM, Kehlet H. Chronic pain as an outcome of surgery. A review of predictive factors. Anesthesiology 2000; 93(4):1123-33. 


\section{Pierides 2016}

Pierides GA, Paajanen HE, Vironen JH. Factors predicting chronic pain after open mesh based inguinal hernia repair. International Journal of Surgery 2016;29:165-70. [DOI: 10.1016/j.ijsu.2016.03.061]

\section{Poobalan 2001}

Poobalan AS, Bruce J, King PM, Chambers WA, Krukowski ZH, Smith WC. Chronic pain and quality of life following open inguinal hernia repair. British Journal of Surgery 2001;88(8):1122-6. [DOI: 10.1046/ j.0007-1323.2001.01828.x]

\section{Poobalan 2003}

Poobalan AS, Bruce J, Smith WC, King PM, Krukowski $\mathrm{ZH}$, Chambers WA. A review of chronic pain after inguinal herniorrhaphy. Clinical Journal of Pain 2003;19(1):48-54.

\section{Rasmussen 2004}

Rasmussen PV, Sindrup SH, Jensen TS, Bach FW. Symptoms and signs in patients with suspected neuropathic pain. Pain 2004;110(1-2):461-9. [DOI: 10.1016/ j.pain.2004.04.034]

Review Manager 2014 [Computer program] The Nordic Cochrane Centre, The Cochrane Collaboration. Review Manager (RevMan 5). Version 5.3. Copenhagen: The Nordic Cochrane Centre, The Cochrane Collaboration, 2014.

\section{Simons 2009}

Simons MP, Aufenacker T, Bay-Nielsen M, Bouillot JL, Campanelli G, Conze J, et al. European Hernia Society guidelines on the treatment of inguinal hernia in adult patients. Hernia 2009;13(4):343-403. [DOI: 10.1007/ s10029-009-0529-7]

\section{Starling 1989}

Starling JR, Harms BA. Diagnosis and treatment of genitofemoral and ilioinguinal neuralgia. World Journal of Surgery 1989;13(5):586-91.

\section{Tasmuth 1996}

Tasmuth T, Estlanderb AM, Kalso E. Effect of present pain and mood on the memory of past postoperative pain in women treated surgically for breast cancer. Pain 1996;68(23):343-7.

\section{Turk 1996}

Turk DC, Okifuji A. Perception of traumatic onset, compensation status, and physical findings: impact on pain severity, emotional distress, and disability in chronic pain patients. Journal of Behavioral Medicine 1996;19(5): 435-53.

\section{Verhagen 2016}

Verhagen T, Loos MJ, Scheltinga MR, Roumen RM. Surgery for chronic inguinodynia following routine herniorrhaphy: beneficial effects on dysejaculation. Hernia 2016;20(1):63-8. [DOI: 10.1007/s10029-015-1410-5]

\section{Werner 2014a}

Werner MU, Bischoff JM. Persistent postsurgical pain: Evidence from breast cancer surgery, groin hernia repair, and lung cancer surgery. Current Topics in Behavioral Neurosciences 2014;20:3-29. [10.1007/7854_2014_285]

\section{Werner 2014b}

Werner MU, Kongsgaard UE. I. Defining persistent postsurgical pain: is an update required?. British Journal of Anaesthesia 2014;113(1):1-4. [DOI: 10.1093/bja/aeu012]

\section{Zwaans 2015} Zwaans WA, Verhagen T, Roumen RM, Scheltinga MR. Factors Determining Outcome After Surgery for Chronic Groin Pain Following a Lichtenstein Hernia Repair. World Journal of Surgery 2015;39(11):2652-62. [DOI: 10.1007/ s00268-015-3183-5]

Zwaans 2017

Zwaans WA, Perquin CW, Loos MJ, Roumen RM, Scheltinga MR. Mesh removal and selective neurectomy for persistent groin pain following Lichtenstein repair. World Journal of Surgery 2017;41(3):701-12. [DOI: 10.1007/ s00268-016-3780-y]

* Indicates the major publication for the study 


\section{A P P E N D I C E S}

\section{Appendix I. Search strategy for MEDLINE (via PubMED)}

(Hernia, Inguinal[Mesh] OR inguinal ${ }^{*}[t i a b]$ OR inguinodyni*[tiab] OR Herniorrhaphy[Mesh] OR herniorrhaph* [tiab] OR hernioplast* [tiab]) AND (((Groin[Mesh] OR groin* [tiab] OR abdominal*[tiab]) AND (Pain[Mesh] OR pain* [tiab])) OR Abdominal Pain[Mesh]) AND (Surgical Procedures, Operative[Mesh] OR "surgery"[Subheading] OR surgical* [tiab] OR surger*[tiab] OR operative* [tiab] OR operation*[tiab] OR mesh[tiab])

\section{CONTRIBUTIONSOFAUTHORS}

All authors contributed equally to the protocol.

\section{DECLARATIONSOF INTEREST}

WARZ: none known; WARZ is a surgical resident and manages patients with (postherniorrhaphy) inguinodynia.

GGK: none known.

KSG: none known; KSG declares funding by the National Institute for Health Research (NIHR) of the United Kingdom, for delivering 30 Cochrane Reviews.

MK: none known; MK is a pain medicine specialist and manages patients with (postherniorrhaphy) inguinodynia.

MRMS: none known; MRMS is a surgeon and operates on patients with (postherniorrhaphy) inguinodynia.

RMHR: none known; RMHR is a surgeon and operates on patients with (postherniorrhaphy) inguinodynia. 